in the bulk liquid, have led us to believe that an exact interpretation of the mechanism involved cannot be obtained without information on the distribution of $\mathrm{He}^{8}$ between vapor and liquid phases below the $\lambda$-point.

Early work ${ }^{1}$ on the abundance ratio of $\mathrm{He}^{3}$ to $\mathrm{He}^{4}$ in the vapor in equilibrium with very dilute solutions of $\mathrm{He}^{3}$ in $\mathrm{He}^{4}$ in the liquid helium II phase showed that this ratio was equal to or less than that in the liquid solution. Subsequent work by Fairbank and Lane ${ }^{4}$ with a similar method has shown that, within the limits of measurement, no $\mathrm{He}^{3}$ exists in the vapor phase below $1.85^{\circ} \mathrm{K}$, when the abundance ratio for the unrefrigerated gas $\mathrm{n}$ as $1.2 \times 10^{-6}$ (atmospheric helium gas). Using a new method in which precautions were taken to assure that any sampling of the vapor would not disturb the liquid-vapor equilibrium and to avoid any possible contamination of the results caused by unwanted film flow, we have measured the equilibrium abundance of $\mathrm{He}^{3}$ between liquid and vapor phases. A description of the apparatus is left to a fuller article. The results are shown in Fig. 1 in which the ordinates give the logarithm of the ratio of the concentration of $\mathrm{He}^{3}$ in $\mathrm{He}^{4}$ in the vapor phase to the concentration in the liquid phase in equilibrium, $\left(\log C_{V} / C_{L}\right)$, i.e., the logarithm of the coefficient of distribution. Above the $\lambda$-point we have plotted in Fig. 1 observations made both by us for atmospheric helium and those previously reported by Lane and collaborators. ${ }^{5}$ Satisfactory agreement is shown. Below the $\lambda$-point we obtain two sets of points which lie roughly along two straight lines, one set for a concentration of the liquid of between 30 to $40 \times 10^{-8}$ (the upper curve) and the other for a liquid concentration of between 9 and $13 \times 10^{-6}$. The variation in the liquid concentration in each set is due to the gradual depletion of the total $\mathrm{He}^{3}$ content of the solution by successive sampling.

It will be seen from the curves that only for the very lowest temperatures and smallest liquid concentrations did the value of the coefficient of distribution fall below unity.

One must conclude that the coefficient of distribution shows a marked variation with concentration, indicating a departure from the laws of perfect solutions even for such dilute solutions as here employed. As yet the variation of the coefficient of distribution with concentration cannot be

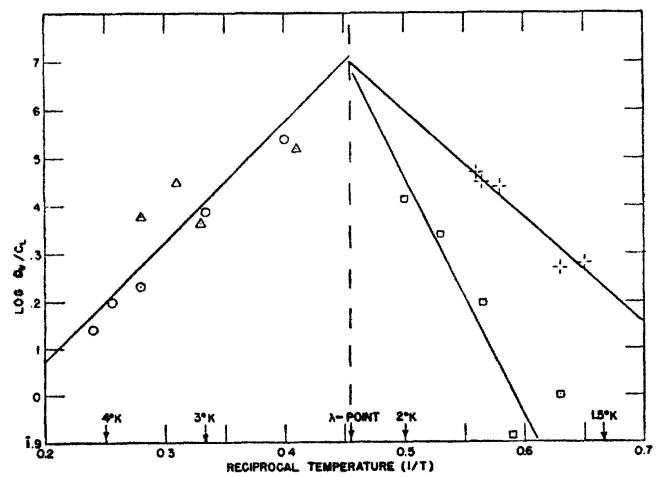

Fig. 1. Variation with temperature of the ratio, in the volume and liquid phases, of the concentration of $\mathrm{He}^{s}$ in $\mathrm{He}^{d}$. The triangles are for concentration $\mathrm{He}^{8} / \mathrm{He}^{4}$ in the liquid phase $=1 \times 10^{-6}$. The squares are for $\mathrm{He}^{\mathrm{s}} / \mathrm{He}^{4}=10 \times 10^{-6}$. The crosses are for Hes/Het
The circles are the results of Lane and collaborators. established, but the general results indicate that with increasing concentration in the liquid solution, relatively more and more $\mathrm{He}^{3}$ goes in to the vapor phase, in agreement with the theory put forward by London and Rice. ${ }^{6}$ Such a departure from ideality was already evident from our measurements ${ }^{7}$ of the osmotic pressures developed in dilute solutions of $\mathrm{He}^{3}$ and $\mathrm{He}^{4}$.

If one applies the thermodynamic treatment of London and Rice, ${ }^{6}$ one obtains for the difference of the enthalpies of condensation of $\mathrm{He}^{3}$ and $\mathrm{He}^{4}: \Delta \bar{H}_{3}-\Delta \bar{H}_{4}=-10$-cal. mole for liquid concentrations of 30 to $40 \times 10^{-6}, \Delta \bar{H}_{3}-\Delta \bar{H}_{4}$ $=-22 \mathrm{cal}$. mole $\mathrm{e}^{-1}$ for liquid concentrations of 9 to $13 \times 10^{-6}$ and for liquid concentrations of $1 \times 10^{-6}$ (not shown in Fig. 1) the value is approximately $-25 \mathrm{cal}^{\mathrm{mole}}{ }^{-1}$. The latter figure, for atmospheric helium, as a result of the increased difficulty of mass spectrographic analysis for small concentrations, has not the same accuracy as the others. These values are a first approximation only since departures from ideality of the solutions have not been taken into account in the calculations; however, the marked difference between these results and those reported elsewhere ${ }^{4}$ remains. Further experimental work on these measurements is being carried out to obtain the function giving the variation of $C_{V} / C_{L}$ with initial concentration, ${ }^{8}$ so that an exact computation can be made of the properties of pure $\mathrm{He}^{3}$.

The results obtained so far, together with those on the osmosis, ${ }^{7}$ have provided sufficient information for a largescale separation process. For such a process, large superfluid flows through supra-surface films can be obtained by increasing the surface by wires ${ }^{9}$ or foils. A technique for this has been outlined by us, ${ }^{10}$ providing a film flow of 0.5 liter liquid per hour, and is being carried out here for continuous separation of $\mathrm{He}^{3}$.

* This work was supported in part by the Office of Naval Research under a contract with the Ohio State Research Foundation. ' J. G. Daunt, R. E. Probst, H. L. Johnston, L. T. Aldrich, and A. O. Nier, Phys. Rev. 72, 502 (1947)

2 J. G. Daunt, R. E. Probst, and H. L. Johnston, J. Chem. Phys. 15, 759 (1947).

${ }^{3}$ C. T. Lane, H. A. Fairbank, L. T. Aldrich, and A. O. Nier, Phys. Rev, 73, 256 (1948).

${ }^{4}$ H. A. Fairbank, C. T. Lane, L. T. Aldrich, and A. O. Nier, Phys. Rev. 73, 729 (1948).

Rev, 71.911 (1947).

6. F. London and Oे. K. Rice, Phys. Rev. 73, 1188 (1948). 1263 (1948).

8 The importance of this is stressed by J.W. Stout in a private communication and is to be published in Phys. Rev.

J. G. Daunt and K. Mendelssohn, Proc. Roy. Soc. A70, 423 (1939).

${ }_{10} \mathrm{~J}$. G. Daunt and H. L. Johnston, The Ohio State University Research Foundation Report to U. S. Naval Office of Research, No. 6 (Jan. 1, 1948).

\section{The Concentration of Helium 3}

Alan ANdrew and W. R. Smythe California Institute of Technology, Pasadena, Californio July 6,1948

$\mathbf{W}$ $\mathrm{E}$ have constructed a two-stage apparatus for concentrating the $\mathrm{He}^{3}$ isotope. The first stage consists of four Clusius-Dickel columns of the concentric tube type connected in parallel. The second stage consists of $29 \mathrm{Hertz}$ pumps connected in series. 
Using the theory of Jones and Furry, ${ }^{1}$ the Clusius-Dickel columns have a calculated performance of 0.036 gram per day concentrated 700 times above normal. The Hertz pumps have a calculated separation factor of approximately $1.8 \times 10^{6}$. The product of these is of the order of $10^{9}$. This is high enough that the final percentage in the Hertz is a linear function of time.

Working with well helium on the basis of continuous operation and using the calculated characteristic time of 7 days, a 0.25 -g product was taken from the first stage and transferred to the second stage where it was processed in 7 days. The first sample of $30 \mathrm{cc}$ at a pressure of $10 \mathrm{~mm}$ had the following analysis:

$\begin{array}{lr}\text { Helium } 3 & 0.5 \text { percent, } \\ \text { Helium } 4 & 59.5 \text { percent, } \\ \text { Hydrogen } & 40.0 \text { percent, }\end{array}$

as analyzed by Consolidated Engineering Corporation of Pasadena on one of their mass spectrometers.

This initial run indicates that nearly a year would be required to produce 50 percent helium 3 ; however, theoretical considerations predict that this time can be materially shortened.

The helium was furnished by the U. S. Navy through Contract N6onr-102, Task Order V.

${ }^{1}$ R. C. Jones and W. H. Furry, Rev. Mod. Phys. 18, 151 (1946).

\section{Fluctuations of Ionization and Low Energy Beta-Spectra}

D. H. W. Kirkwood, B. Pontecorvo, and G. C. Hanna National Research Council. Chalk River Laboratory. Chalk River, Ontario, Canad June 29, 1948

TSING an $(n, \gamma)$ reaction in the Chalk River heavy water pile, we have prepared the nuclide $\mathrm{A}^{37}$, which is known to decay by $K$-capture without emission of positrons. ${ }^{1} \mathrm{~A}^{37}$ was introduced inside various proportional counters filled with a mixture of argon and methane: usually, though not always, $39 \mathrm{~cm} A+12 \mathrm{~cm} \mathrm{CH}_{4}$ were used. The cathode diameters, wire diameters, and effective lengths of two typical counters, 1 and 2, were, respectively, $4.67 \mathrm{~cm}, 0.0047 \mathrm{~cm}, 19.7 \mathrm{~cm}$ for No. 1 and $1.75 \mathrm{~cm}, 0.0047$ $\mathrm{cm}, 6.9 \mathrm{~cm}$ for No. 2. The counters were connected to a linear amplifier, the output of which was fed either into a discriminator or into a 30-channel pulse analyzer. A typical differential curve is shown in Fig. 1, obtained with counter 1 working at 2520 volts. The noise was equivalent to 4500 pair of ions and the multiplication factor in this run was about $10^{4}$. The curve is shown by a broken line below channel 2, because the effective width of channel 1 is not accurately known. The main peak corresponds to an energy of about $2800 \mathrm{ev}$, the binding energy of a $K$ electron in the $\mathrm{Cl}^{37}$ atom. Since the Auger electron yield ${ }^{2}$ for the $K$ shell is about 90 percent, about 90 percent of the $K$-capture processes result in the emission of soft radiations dissipating the full $2800 \mathrm{ev}$ in the counter.

The low energy peak corresponds to about $200 \mathrm{ev}$, as was

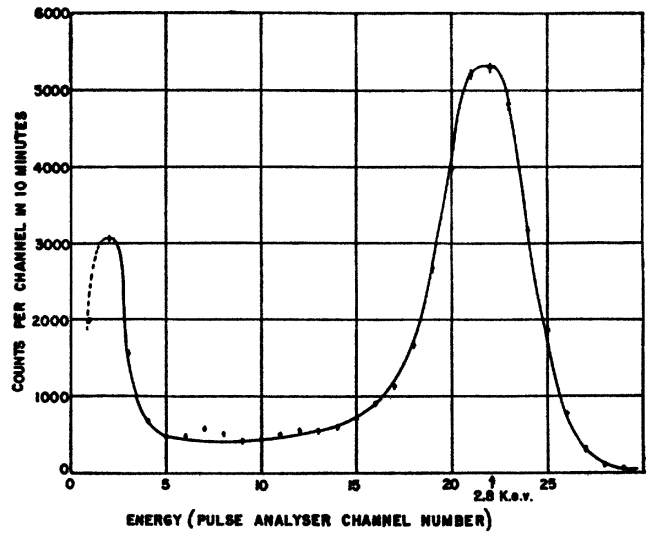

Fig. 1.

determined by runs at higher over-all gain. This is approximately the energy of ionization of the $L$ shell. We believe that the following two mechanisms contribute in a comparable extent to the low energy peak: (a) Nuclear $K$-capture processes resulting in the $K-\alpha$-x-radiation being emitted by the $\mathrm{Cl}^{37}$ atom and not being absorbed in the counter. In such processes a photon of about $2600 \mathrm{ev}$ would escape from the counter, while the remaining energy corresponding to the ionization of the $L$ shell would be dissipated in the counter. (b) Nuclear absorption of an $L$ electron, the so-called $L$-capture, which has been discussed in the literature but not yet observed experimentally. ${ }^{3}$ While we plan to discuss in the near future the quantitative aspects of this question, we wish to turn now to the main peak.

1. While the main peak cut-off is very sharp on the high energy side, there is a "tail" on the other side. That this is partly due to the end effects of the counter, resulting in a small value of the multiplication factor near the end, has been shown by a few runs recently obtained with a counter (3) having a ratio of length to diameter three times as great as the one used in the run illustrated in Fig. 1. With counter 3 the cut-off on the low energy side is sharper.

2. That the mean energy necessary for an electron to produce a pair of ions is approximately independent of the electron energy was confirmed by experiments in which the $K-\alpha$-x-ray lines of copper were made to fall upon the counter. Although there was a wide distribution of pulses as a result of wall effects, the maximum size, caused by the full energy content of the copper $K-\alpha$-quantum $(\approx 8000 \mathrm{ev})$ could be measured on an oscilloscope. The ratio of this maximum pulse size to the main $\mathrm{A}^{37}$ pulse size was $2.8 \approx 8000 / 2800$. It is clear that $\mathrm{A}^{37}$ can provide an ideal "calibration line" for the proportional counter investigation of low energy beta-spectra such as that of $\mathrm{H}^{3}$.

3. The width at 60.7 percent of the peak for the $2800-\mathrm{ev}$ line is about 18 percent as determined in various runs. This corresponds to an observed standard deviation of the pulse size of \pm 9 percent. Yet the mean number of pairs of ions produced by a 2800 -ev electron is about 100 , and if we assume that the variance of the number of 